\title{
Event-Related Bias Removal for Real-time Disaster Events
}

\author{
Evangelia Spiliopoulou* Salvador Medina Maza* Eduard Hovy Alexander Hauptmann \\ Language Technologies Institute \\ Carnegie Mellon University \\ \{espiliop, salvadom, ehovy, alex\}ecs.cmu.edu
}

\begin{abstract}
Social media has become an important tool to share information about crisis events such as natural disasters and mass attacks. Detecting actionable posts that contain useful information requires rapid analysis of huge volume of data in real-time. This poses a complex problem due to the large amount of posts that do not contain any actionable information. Furthermore, the classification of information in real-time systems requires training on outof-domain data, as we do not have any data from a new emerging crisis. Prior work focuses on models pre-trained on similar event types. However, those models capture unnecessary event-specific biases, like the location of the event, which affect the generalizability and performance of the classifiers on new unseen data from an emerging new event. In our work, we train an adversarial neural model to remove latent event-specific biases and improve the performance on tweet importance classification.
\end{abstract}

\section{Introduction}

Effective management of crisis situations like natural disasters (e.g. earthquakes, floods) or attacks (e.g. bombings, shootings) is an extremely sensitive and complex phenomenon that requires efficient coordination of people from multiple disciplines along with proper allocation of time and resources (Tapia et al., 2011a; Maitland et al., 2009). Given that we live in the era of information and social media, filtering important nuggets of information from real-time data and using them into decision-making constitutes a crucial research direction (Tapia et al., 2011b).

Critical information from social media is found only in small amounts. Hence it is difficult to ex-

\footnotetext{
* Equal contribution.
}

tract and analyze the data stream, since it is impossible to manually process the amount of information shared in social media in real-time. Therefore, it is important to detect data that contain useful information for decision-making and automatically extract it (Sutton et al., 2008; Palen et al., 2010). Even though sentence classification is a well-studied NLP problem, common approaches do not bring the expected results (Reuter et al., 2018).

The main reason why common approaches fail is the lack of in-domain data (Mccreadie et al., 2019; Hiltz et al., 2014). Most emerging crisis are unexpected and data analysis must be done real-time, within a small time-frame (Plotnick et al., 2015). Even if we might have high quality annotated data from previous similar crisis situations, we will not have data from the emerging event that we want to classify. For example, let us assume an earthquake in Seattle happens right now. Although we may have annotated data from a previous earthquake in Los Angeles, most of the parameters would be entirely different (e.g. location names, damages, times, etc) since the cities and populations differ. Furthermore, because some of those parameters might indeed play an important role in the classification of a tweet from the specific event (e.g. location, if Monroe is the epicenter of the Seattle earthquake), a traditional model would learn them as important features. This creates a highly-biased model that does not generalize on future events, since we cannot fine-tune properly on-the-fly. On the other hand, some other features are actually important in the general setting (e.g. severity of the earthquake, casualties etc.). The problem we tackle in this work is how to construct an event-based zeroshot learning model that can learn unbiased representations, instead of relying on a highly-biased set of features from seen data.

In this paper we explore a technique that helps a neural model to distinguish and discard informa- 
tion that is related only to specific events, resulting in a more generalizable model with improved performance on unseen events without any fine-tuning. Since the main task is to classify the importance of the information contained in a tweet (criticality), we use an adversarial classifier that intends to learn which specific event the tweet refers to, hence remove the event specific bias through a reversal gradient. Our experiments represent a reallife crisis management scenario, where the model is evaluated on a new incoming event through a leaveone-out experimental setup, and show substantial improvement over baseline classification methods. Finally, we share our code for reproducibility and ease of use ${ }^{1}$.

\section{Related Work}

Recent work on crisis informatics focuses on developing NLP solutions to classify and extract information from Twitter streams and other social media data related to an emergency event (e.g. attacks, natural disasters). As discussed by Tapia et al. (2011b), there are several problems under the umbrella of crisis informatics, such as determining if a snippet of text is related to a specific event, if it is reliable and trustworthy, the type of information it contains, whether the information is actionable, etc. Most previous work focuses on the relevance problem: given a set of tweets or other source of information and a specific event, classify which data refer to that event. Caragea et al. (2016) uses a CNN model to classify tweets related to flood events, while Kruspe (2019) uses a few-shot learning model based on a CNN. Nguyen et al. (2016) also uses a CNN model to classify related tweets and the type of information contained (e.g. infrastructure damage, affected individuals etc) from the Nepal 2015 earthquake. Neubig et al. (2011) introduces a real-time system for the Japan 2011 earthquake that classifies the relatedness of the posts and extracts surface information like named entities. Other approaches include BiLSTM models for tweet classification (Ma), event detection based on Twitter streams (Sakaki et al., 2010), adversarial data augmentation for image classification (Pouyanfar et al., 2019) and domain-adaptation across different events using an adversarial network.

It is particularly important to first responders the identification of actionable information from a stream of messages as the one provided by Twitter.

\footnotetext{
${ }^{1}$ https://salmedina.github.io/EventBiasRemoval/
}

Munro (2011) proposes a system based on a set of features (location, time, n-grams) to label text messages as actionable/ non-actionable. Most recently, the TREC-IS challenge by Mccreadie et al. (2019) proposes a labeling scheme where the actionability of a tweet is replaced by the information type and the criticality score. Higher criticality indicates a post contains more relevant information that could be useful for public safety officers during an emergency. Although Miyazaki et al. (2019) shows a great improvement on information type extraction by using Bi-LSTM attention on BERT embeddings, identifying critical and actionable information is a much harder task (Mccreadie et al., 2019).

Processing information without the context of a crisis event is a bottleneck for big data crisis analytics, as discussed by Qadir et al. (2016). The lack of context makes the classification of messages very difficult, since the models are prone to eventspecific biases. Due to the fact that we deal with real-time data, a domain-adaptation approach cannot use fine-tuning in a zero-shot scenario, which results in highly-biased models. Most recent work on bias removal (Elazar and Goldberg, 2018) focuses on using adversarial learning to remove demographic bias from representations. Examples include adversarial generative networks that create fair representations (Madras et al., 2018), metrics to quantify unintended biases (Borkan et al., 2019) and applications that show substantial improvements on traditional NLP tasks like NLI (Lu et al., 2018), Coreference Resolution (Belinkov et al., 2019) and text classification (Zhang et al., 2018) by using unbiased representations. Our approach is inspired by the work of Elazar and Goldberg (2018) on bias removal through an adversarial attack. The authors use an adversarial setting to remove demographic information from text and construct cleaner representations. In our case, the adversarial classifier attempts to predict the event to which the tweet belongs. Another difference with our work is the imbalanced data used for training the classifier of the main task. Other related work includes domain adaptation based on a gradientreversal layer (Ganin et al., 2016), text classification based on adversarial multi-task learning (Liu et al., 2017), and multi-adversarial domain adaptation across multi-modal data (Pei et al., 2018). 

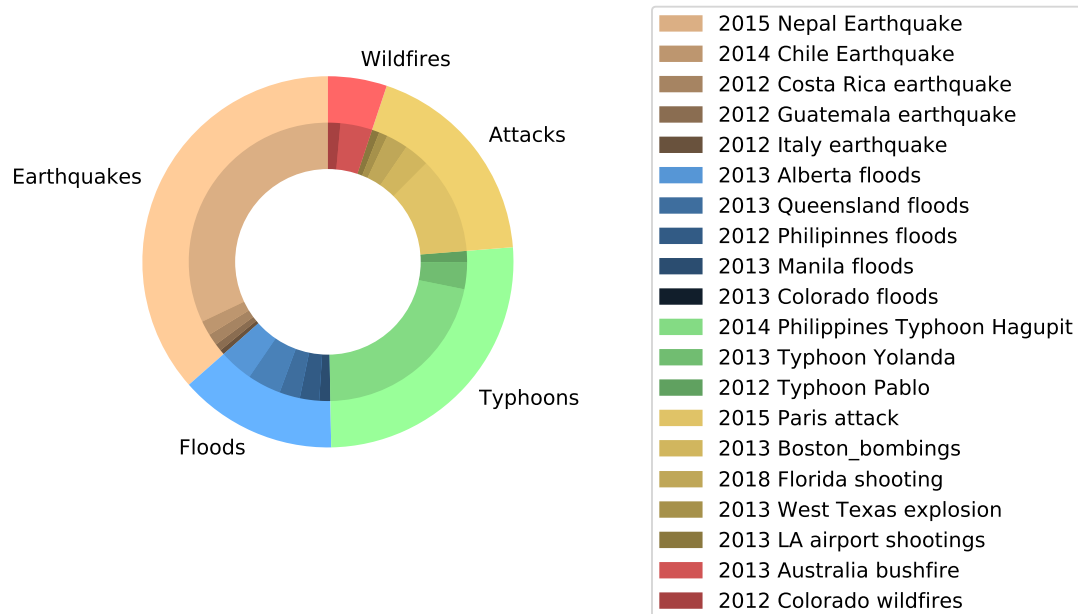

Figure 1: Crisis NLP Dataset Distribution. Outer circle: Color defines each of the event categories. Inner circle: The shade of colors describe the different events within a category.

\section{Approach}

In this work we used data from the TREC 2018 Incident Streams challenge ${ }^{2}$, which contains labels on criticality and information types (Mccreadie et al., 2019). They define criticality as a score to identify posts that need to be shown to an officer immediately as an alert. The raw data and information about the specific event each tweet belongs to is extracted from the Crisis NLP (Imran et al., 2016) dataset, which contains tweets in English from disaster events that occurred during 2012-2018. The crisis events in our dataset can be split into five main groups: earthquakes, floods, typhoons, wildfires and attacks. In Figure 1, we show that the data mainly consists of multiple earthquake, flood, and typhoon events, only two wildfire events, and five diverse attacks originated by humans.

\subsection{Data Description}

Table 1: Examples of Critical and Non-Critical Tweets

\begin{tabular}{lll}
\hline Label & Event & Tweet \\
\hline non-critical & 2014 Philippines Typhoon & $\begin{array}{l}\text { Good morning! keep safe everyone! } \\
\text { RT: Seek higher ground immediately } \\
\text { wall of water coming down Boulder Canyon } \\
\text { move away from Boulder Creek }\end{array}$ \\
critical & 2013 Colorado Floods & $\begin{array}{l}\text { I am honestly sick who could be so } \\
\text { disgusting to do this to someone we will get } \\
\text { answers and find you \#prayforboston }\end{array}$ \\
non-critical & 2013 Boston Bombings & $\begin{array}{l}\text { RT: News at epicenter of Nepal tragedy } \\
\text { local church mission offers help! }\end{array}$ \\
\hline
\end{tabular}

In our experiments we used a labeled subset of the data formed by 18,283 tweets which are labeled into four categories according to their level of importance for the authorities: low, medium,

high, and critical. The distribution of the labels is highly skewed towards the low and medium labels as shown in Figure 2a. These types of tweets do not provide important information for decision-making during a disaster event. Since we are aiming to sieve the actionable tweets, we grouped together the low and medium labels as non-critical, and the high and critical as critical. The new distribution of the data after relabeling is shown in Figure 2b. As we see on the examples shown in Table 1, the latter have actionable information for the authorities, first responders, and population on distress.

\subsection{Data Pre-processing}

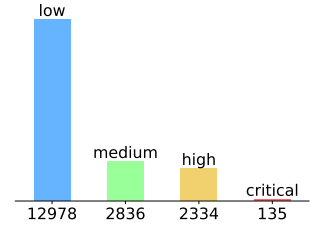

(a) Original labels

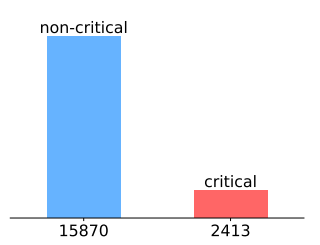

(b) New labels

Figure 2: Dataset label distribution. (a) Label distribution of original dataset, (b) Distribution of the labels after grouping $\{$ low, medium $\}$ as non-critical and $\{$ high, critical $\}$ as critical

Our target dataset comes from Twitter. Therefore, we performed a series of pre-processing steps for data-cleaning. First, we removed links, hashtags and mentions, since most of them are event specific. We also removed non-English words to reduce the noise. Next, we removed all non-English

\footnotetext{
${ }^{2}$ http://dcs.gla.ac.uk/richardm/TREC_IS/2020/oldindex.htmlcharacters and emojis. Finally, we observed that
} 
many times white spaces were omitted between words, which resulted in multiple words being clustered as a single token. To solve that, we stripped the text from punctuation marks and, subsequently, used a heuristic for word segmentation, where we split the token into the least number of possible English words via greedy search.

\subsection{Models}

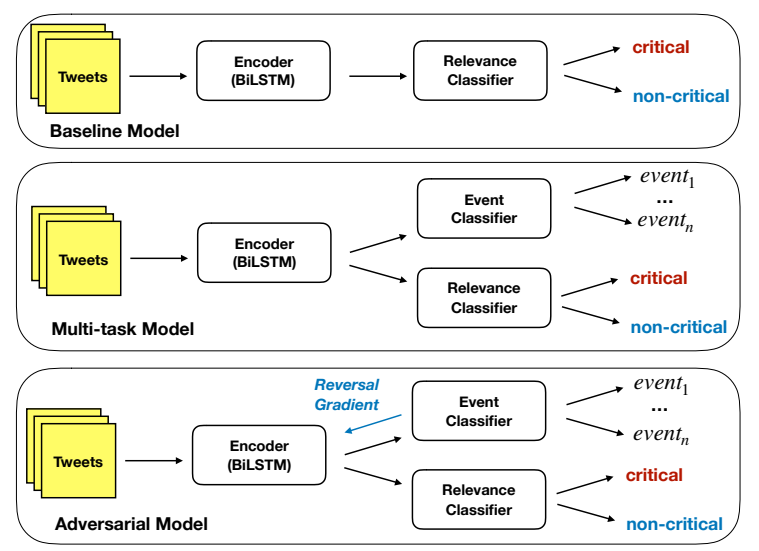

Figure 3: Evaluated Model Architectures. The adversarial model was compared against the baseline and multitask models to show the removal of event specific biases.

Our experimental setup consists of a dataset $\mathcal{D}$ composed of tweets $t_{1}, \ldots, t_{n}$ and two sets of labels; $y_{e_{1}}, \ldots, y_{e_{n}}$ representing the event that the tweet belongs to and $y_{r_{1}}, \ldots, y_{r_{n}}$ representing the importance of the tweet, where $y_{r_{i}} \in$ $\{$ non-critical,critical $\}$. For this task we want to find the optimal classifier $f$ for predicting labels $y_{r_{i}}$. In this work we compared three models to measure if an adversarial training contributes to the detection of critical tweets on unseen events.

Our main hypothesis states that an adversarially trained model removes event-specific information, while focusing on features that determine how important the tweet is. For our experiments we compare the adversarially trained model against a binary classifier and a multi-task model. The comparison between the multitask and the adversarial models helps us evaluate whether the explicit removal of bias-related information benefits the relevance classifier or if using a model that jointly learns both tasks suffices.

\subsubsection{Baseline Model}

In our baseline model setup, a tweet $t_{i}$ is a sequence of word embeddings $w_{1}, \ldots, w_{m_{i}}$ which are encoded through an LSTM (Graves et al., 2013) encoder $h$. Then the generated embedding $h\left(t_{i}\right)$ is fed to a binary classifier $c_{r}$ that learns to predict if the tweet is critical or non-critical. The architecture of this model is shown in Figure 3.

The training loss $\mathcal{L}$ used across all the models and experiments is cross-entropy. The optimization of the baseline model is described in eq. 1 .

$$
\underset{h, c_{r}}{\operatorname{argmin}} \mathcal{L}\left(c_{r}\left(h\left(t_{i}\right)\right), y_{r i}\right)
$$

\subsubsection{Multi-task Model}

The multitask learning setup described by Caruana (1997) aims to improve the performance of a model by learning multiple tasks at the same time. Since the dataset is divided per disaster event, we take advantage of this information given by the structure of the dataset, and define event detection as the second learning task along with the criticality classification. Hence, the multitask model adds an event classifier $c_{e}$ on the encoding of the incoming tweet $h\left(t_{i}\right)$ which trains simultaneously with the classifier $c_{r}$, as seen in Figure 3.

The optimization procedure for this model is described in eq. 2 .

$$
\underset{h, c_{r}, c_{e}}{\operatorname{argmin}} \mathcal{L}\left(c_{r}\left(h\left(t_{i}\right)\right), y_{r i}\right)+\mathcal{L}\left(c_{e}\left(h\left(t_{i}\right)\right), y_{e i}\right)
$$

\subsubsection{Adversarial Model}

The adversarial model used in this work follows the adversarial training setup proposed by Goodfellow et al. (2014), Ganin et al. (2016), and Xie et al. (2017). In essence, the adversarial model is similar to the multitask model except for the addition of a gradient-reversal layer $g_{\lambda}$ (Ganin et al., 2016) between the encoder $h$ and the event classifier $c_{e}$. The gradient-reversal layer during a forward step works as the identity function $\mathcal{I}$, but during the back-propagation step the gradient from $c_{e}$ is reversed and scaled by a value $\lambda$. In our work, we intend to achieve domain adaptation from previous events to a new incoming event by minimizing the information related to previously seen events provided by $c_{e}$, while maximizing the information gain obtained from classifier $c_{r}$, as described in eq. 3.

$$
\underset{h, c_{r}, c_{e}}{\operatorname{argmin}} \mathcal{L}\left(c_{r}\left(h\left(t_{i}\right)\right), y_{r i}\right)+\mathcal{L}\left(c_{e}\left(g_{\lambda}\left(h\left(t_{i}\right)\right)\right), y_{e i}\right)
$$




\section{Experiments}

For our experiments we used two of the main popular word embeddings to represent the tokens of the tweets in the target dataset: GloVe (Pennington et al., 2014) embeddings, and BERT (Devlin et al., 2019) embeddings.

We used the 100-dimensional GloVe embeddings pre-trained on Wikipedia and Gigaword, which were made publicly available by the authors $^{3}$. For extracting BERT embeddings we used the Python package bert-embeddings ${ }^{4}$ as we built the networks for our experiments in PyTorch. This package offers a pre-trained 768-dimensional hidden state transformer model with 12-layers and 12-headed attention. In our experiments, the BERT model was frozen with no fine-tuning during training.

Throughout all of our experiments the tweet encoder $h$ is an LSTM with two layers. Each of the LSTMs have a hidden dimension of 100, which results in a tweet embedding of size 200. Both classifiers $c_{r}$ and $c_{e}$ are linear layers with output size 2 and the number of events per experiment, respectively. During our initial experimentation, we set the gradient-reversal layer scaling value lambda to different values within the range $[0.1-10]$. The most stable result throughout the whole experiments was obtained with $\lambda=1$.

The models were trained using the Adam optimizer (Kingma and $\mathrm{Ba}, 2014$ ), with an initial learning rate 0.01 , batch size 16 and trained for 40 epochs. We employed dynamic batching by padding each batch to the sequence length of the longest sample in the batch.

To test the performance of the model at every epoch we calculated the micro F1 on the critical class from $c_{r}$ and considered as the best model the one which showed the highest Critical-F1 score, since for disasters it is important to recall as many critical tweets with the highest possible precision.

\subsection{Model Evaluation}

Since we intend to evaluate the models for a reallife scenario, we used data from each disaster type separately (e.g. model trained and tested only on flood events), to perform an analysis in a disasterbased zero-shot learning scenario simulating an incoming unseen event. To achieve this, the training

\footnotetext{
${ }^{3}$ https://nlp.stanford.edu/projects/glove/

${ }^{4}$ https://github.com/imgarylai/bert-embedding
}

data consists of all the events of the same disaster type except one, as it is used for testing the model. We generated $n$ splits for each event type, where $n$ is the amount of events per event type. We evaluated the three models on each split obtaining the macro-F1 and the micro-F1 scores from the $c_{r}$ predictions. Finally, we calculated the mean of these metrics, which we can see in Table 2. The best models for each event type are highlighted in the representative color of the event, as shown in Figure 1.

Since we follow a leave-one-out testing procedure, we could not include the wildfires event type since this category only has two instances. This makes it impossible to train the multitask and adversarial models on this type of event.

Our experiments show an improvement of the F1 score for all disaster events that use adversarial training except for the attacks group, where the improvement is not consistent with the rest of the events. The earthquake and flood events show a significantly better performance of the adversarial model when compared to both the baseline and the multitask model. For the typhoon events the multitask model improves slightly over the baseline, but the adversarial model is the best for both embedding types, while BERT has better results than GloVe by a large margin.

Most similar to our setting, Nguyen et al. (2016) performs an experiment in an online training scenario using the Nepal 2015 Earthquake as test set, while more than 10,000 tweets from the dataset are used for pre-training the model. Their work reports an AUC of 0.73 at the beginning of the event, which would be comparable to our zero-shot learning scenario. To compare our model to their work, we used the data split where the Nepal earthquake was left out for testing the model. On this data split, the adversarial model using BERT embeddings obtains an AUC of 0.62 for the critical class while training with only 815 tweets from all the other earthquake events.

\subsection{Event Types Data Mix}

In Figure 1, we observe that the attack events group consists of diverse types of events such as shootings, bombings, and explosions. Even though all of those events contain violence-related incidents, the adversarial model with BERT embeddings has lower performance than the baseline and the multitask learning model, as shown in the results on 
Table 2: Event based zero-shot test results. The best model per disaster type is highlighted with the color assigned to the disaster type. The best model per embedding type is highlighted in bold.

\begin{tabular}{|c|c|c|c|c|c|}
\hline Event Type & Embedding & Model & $\begin{array}{l}\text { Macro } \\
\text { F1 }\end{array}$ & $\begin{array}{c}\text { Non-Critical } \\
\text { F1 }\end{array}$ & $\begin{array}{c}\text { Critical } \\
\text { F1 }\end{array}$ \\
\hline \multirow{6}{*}{ Earthquakes } & \multirow{3}{*}{ GloVe } & Baseline & 0.6432 & 0.9082 & 0.3782 \\
\hline & & Multitask & 0.5890 & 0.8960 & 0.2819 \\
\hline & & Adversarial & 0.6602 & 0.9170 & 0.4034 \\
\hline & \multirow{3}{*}{ BERT } & Baseline & 0.6138 & 0.9062 & 0.3213 \\
\hline & & Multitask & 0.5844 & 0.8863 & 0.2826 \\
\hline & & Adversarial & 0.6154 & 0.8888 & 0.3420 \\
\hline \multirow{6}{*}{ Floods } & \multirow{3}{*}{ GloVe } & Baseline & 0.6010 & 0.8674 & 0.3346 \\
\hline & & Multitask & 0.6130 & 0.8679 & 0.3581 \\
\hline & & Adversarial & 0.6326 & 0.8454 & 0.4198 \\
\hline & \multirow{3}{*}{ BERT } & Baseline & 0.6145 & 0.8834 & 0.3455 \\
\hline & & Mulitask & 0.6062 & 0.8793 & 0.3331 \\
\hline & & Adversarial & 0.6403 & 0.8642 & 0.4164 \\
\hline \multirow{6}{*}{ Typhoons } & \multirow{3}{*}{ GloVe } & Baseline & 0.5714 & 0.8965 & 0.2462 \\
\hline & & Multitask & 0.5832 & 0.8961 & 0.2702 \\
\hline & & Adversarial & 0.5887 & 0.8916 & 0.2858 \\
\hline & \multirow{3}{*}{ BERT } & Baseline & 0.6249 & 0.9189 & 0.3310 \\
\hline & & Mulitask & 0.6291 & 0.9091 & 0.3491 \\
\hline & & Adversarial & 0.6302 & 0.9086 & 0.3517 \\
\hline \multirow{6}{*}{ Attacks } & \multirow{3}{*}{ GloVe } & Baseline & 0.6049 & 0.9047 & 0.3052 \\
\hline & & Multitask & 0.5994 & 0.8917 & 0.3071 \\
\hline & & Adversarial & 0.6056 & 0.8975 & 0.3137 \\
\hline & \multirow{3}{*}{ BERT } & Baseline & 0.5744 & 0.8840 & 0.2649 \\
\hline & & Multitask & 0.6165 & 0.9009 & 0.3322 \\
\hline & & Adversarial & 0.5492 & 0.8511 & 0.2472 \\
\hline
\end{tabular}

Table 3: Mixed flood and typhoon test results

\begin{tabular}{lccc}
\hline Model & Macro F1 & Non-Critical F1 & Critical F1 \\
\hline Baseline - GloVe & $\mathbf{0 . 5 3 7 6}$ & 0.7602 & 0.3150 \\
MultiTask - GloVe & 0.5331 & 0.7529 & 0.3133 \\
Adversarial - GloVe & 0.5157 & 0.7428 & 0.2885 \\
\hline Baseline - BERT & 0.5593 & 0.7602 & 0.3584 \\
MultiTask - BERT & $\mathbf{0 . 5 6 2 5}$ & 0.7558 & 0.3692 \\
Adversarial - BERT & 0.5539 & 0.7500 & 0.3578 \\
\hline
\end{tabular}

Table 2. Our hypothesis is that the adversarial model fails to remove the event-specific biases in the Attack group, because of the mixture of different event types. A potential solution to this problem would be to include more events to facilitate the disentanglement of the Attacks group.

To test this hypothesis, we created a synthetic event type where we mix flood and typhoon events, since both are disasters that would result in flooded cities and towns. We repeated the same experimental procedure by leaving out one event for testing and obtained the mean scores across all splits, as reported in Table 3. The results from this experiment verify our hypothesis that the adversarial training of the classifier is sensitive to the entanglement of events in the training data. This supports our claim on why we have low performance on attacks and highlights the importance of not mixing different event types when training under an adversarial setup.

\section{Qualitative Analysis}

We took a deeper look into our experimental results by comparing which patterns are learnt by the adversarial model but not the baseline. For this analysis, we focused on flood and earthquake event types, as they show the greatest difference in 
Table 4: Examples captured by the adversarial model (true-positives), but not the baseline (false-negatives).

\begin{tabular}{|c|c|}
\hline True Label & Tweet Text \\
\hline Critical & $\begin{array}{l}\text { rt flood in the ust hospital is now on the } 2 \text { nd floor } \\
\text { no food for the patients \& staff pls help ... }\end{array}$ \\
\hline Critical & rt please help rt rt those who are in $\mathrm{u}$ erm the flood is now goi ... \\
\hline Critical & $\begin{array}{l}\text { ust hospital and u erm in need of immediate help u sts } \\
\text { morgue is flooded ue rms nursery is near being flooded please please }\end{array}$ \\
\hline Critical & philippine flood fatalities hit 23 \\
\hline Non-Critical & $\begin{array}{l}\text { metro manila flood updates nlex is now north luzon express river } \\
\text { pls rt and spread }\end{array}$ \\
\hline Non-Critical & $\begin{array}{l}\text { ndr rmc nearly } 50 \text { of metro manila submerged in floodwater } \\
\text { due to heavy monsoon rains }\end{array}$ \\
\hline Non-Critical & $\begin{array}{l}\text { rt lets all pray for those who lost their homes and now living in } \\
\text { cold and starving ... }\end{array}$ \\
\hline Non-Critical & $\begin{array}{l}\text { rt pal passengers to/from manila who are unable to take } \\
\text { their flights due to floods may rebook their tickets with rebooking } \mathrm{c} . .\end{array}$ \\
\hline
\end{tabular}

Table 5: Test results on 2012 Philippines Flood

\begin{tabular}{lccc}
\hline Model & Macro F1 & Non-Critical F1 & Critical F1 \\
\hline Baseline - BERT & 0.5844 & 0.8413 & 0.3274 \\
MultiTask - BERT & 0.5875 & 0.8766 & 0.2985 \\
Adversarial - BERT & $\mathbf{0 . 6 5 3 5}$ & 0.8832 & $\mathbf{0 . 4 2 3 8}$ \\
\hline
\end{tabular}

F1 score between the baseline and the adversarial model.

\subsection{Critical Detection Comparison}

For the first part of the qualitative analysis, we examined tweets where the baseline and the adversarial models disagree upon. We looked at both critical and non-critical tweets in order to find common patterns where the models fail. In Table 4 we show some examples of tweets where the baseline model failed, but were correctly classified by the adversarial model. The examples used come from the Philippines flood (performance shown in Table 5).

A consistent pattern observed for the critical tweets is that they mostly contain information about a need for emergent help or a situation currently happening. Furthermore, we see a strong sentiment of despair, where we may assume that the users are directly affected by the event. On the other hand, if we look at the non-critical tweets that were incorrectly classified as critical by the baseline, they mostly contain location information and named entities. As mentioned earlier, in a zero-shot scenario upon the development of a crisis event, the mod- els trained on previous similar scenarios perform poorly due to event bias found in the data. Through those examples we see that our approach successfully removes part of that bias through adversarial learning.

\subsection{Model Comparison via Saliency Maps}

For the second part of our analysis, we used saliency maps to visualize the relevance of each word in a tweet for the models. We selected tweets that contain named entities (e.g. locations, names) or information that is generally important to classify a tweet, such as casualties. For this part, we only used GloVe embeddings, since BERT is context-based and each embedding may encode information from the rest of the tweet.

In order to construct the saliency map, we used back-propagation to estimate the first-order derivatives from each word, as a measure of their contribution to the model's decision. This strategy was adopted from the vision community (Erhan et al., 2009; Simonyan et al., 2013), and recently adapted in NLP research (Li et al., 2016).

In Figure 4 we visualize the saliency map of each word embedding for the baseline and adversarial models. The higher the absolute value of the first-order derivative (dark blue and white), the more important role it plays into the classifier's decision. We observe that, for the first and second sentences, the baseline puts more weight on the location, which is a strong event-bias since it includes information only for a particular event and 

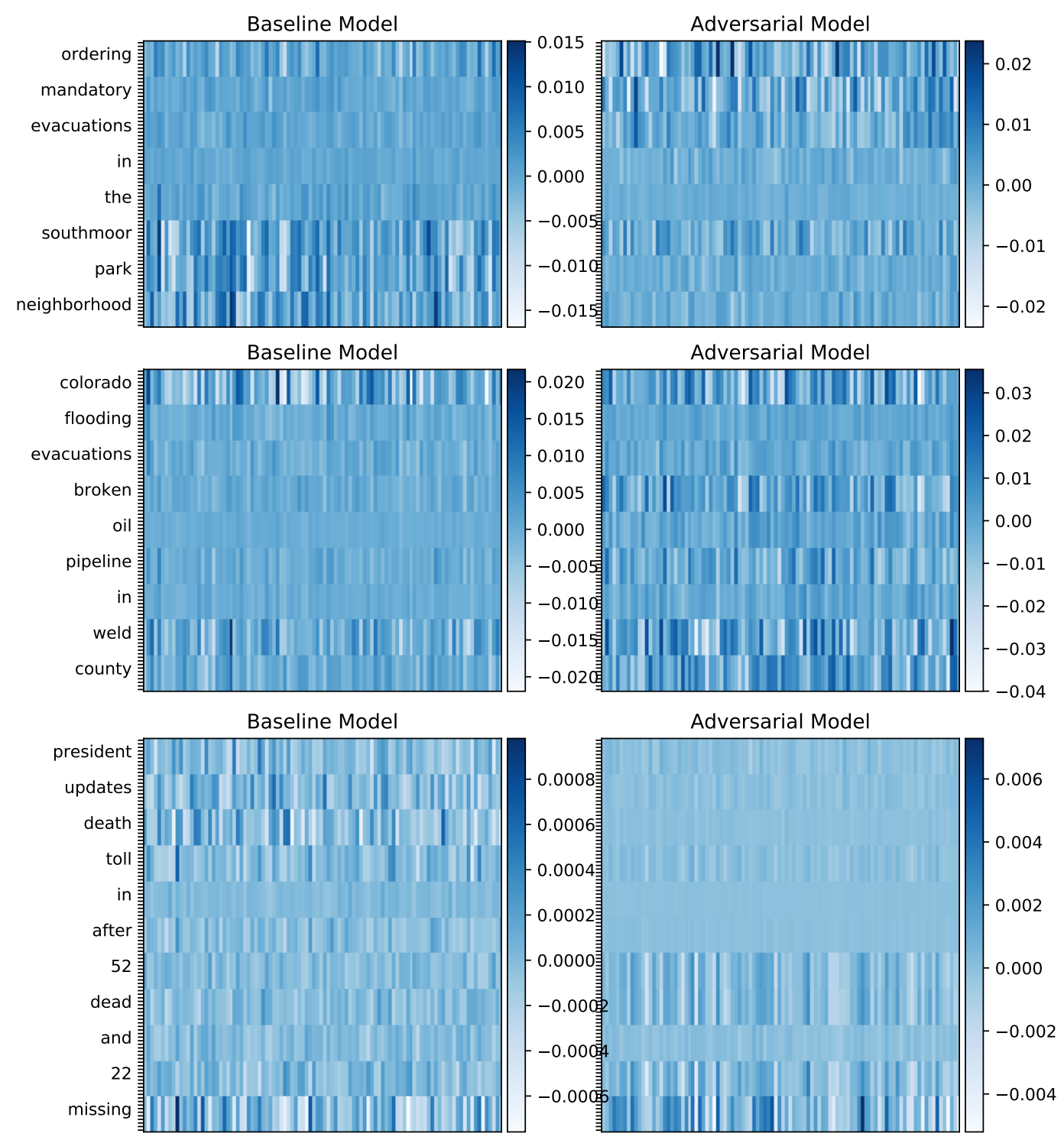

Figure 4: Saliency map visualization of tweets with strong event-bias.

not a disaster type (e.g. floods). On the other hand, the adversarial model focuses more on important sub-events, like mandatory evacuations and broken pipeline, which we desire to capture in a zero-shot scenario, and is generally ignored by the baseline model. We further observe a similar trend for the third sentence, where the baseline gives mostly uniform weight with a small focus on president updates death, while the adversarial model focuses more on generally informative text that describes casualties.

\section{Future Work}

Our experiments show that mixing data from events whose semantics are similar, like the violent mass attacks and the synthetically generated set of floods and typhoons, confuses the adversarial model. As a result, it does not show any improvement over the baseline. Moreover, in some cases models trained with GloVe achieved better performance compared to those trained with BERT. For this reason, it seems appropriate to fine-tune transformer-based language models so we could take advantage of the large amount of unlabeled data provided by the Crisis NLP dataset that was not used in this work.

Given that our ultimate goal is to detect and use actionable information during crisis events to inform life-saving actions, an essential part of future research is to design interpretable models. An interesting work proposes a new approach to interpretable classification named deep weighted averaging classifiers (DWAC) (Card et al., 2019), which gives an explanation of the prediction in terms of the weighted sum of training instances. DWAC 
could replace the importance classifier $c_{r}$ in our proposed adversarial model. An advantage of using DWAC is that it would deliver the most relevant tweets from the training data which contributed to the detection of a critical tweet.

Finally, since we deal with a real-time information stream it seems appropriate to evaluate this model in an online learning scenario (Nguyen et al., 2016).

\section{Conclusion}

In this work, we compared an adversarialy trained model against a baseline classifier and a multitask learning model. The main task for all the models was to predict if a tweet is critical or non-critical over four types of disaster events: earthquakes, floods, typhoons, and mass attacks in public spaces. We presented a thorough analysis on how a simple classification model trained on crisis event data can be improved through adversarial training. Our results showed how the addition of an adversarial network removes the bias from specific events, allowing the network to put more attention in disaster related information rather than specificities of a particular event. In most of our experiments the adversarially trained model obtained the highest F1 score.

Our experimental results demonstrate the relevance of using micro-F1 scores for evaluating the detection of critical posts from an information stream such as Twitter. The impact of false negatives while detecting critical tweets is larger than the false positives, since we would be missing decisive information from the data stream. Hence, micro-F1 score is a more informative metric to consider instead of accuracy, or even the overall F1 score since event crisis detection usually suffers from highly skewed data towards the irrelevant samples of the dataset.

\section{Acknowledgments}

This research was partially supported by DARPA grant no HR001117S0017-World-Mod-FP-036 funded under the World Modelers program, as well as by the financial assistance award 60NANB17D156 from U.S. Department of Commerce, National Institute of Standards and Technology (NIST). The U.S. Government is authorized to reproduce and distribute reprints for Governmental purposes notwithstanding any copyright annotation/herein. Disclaimer: The views and con- clusions contained herein are those of the authors and should not be interpreted as necessarily representing the official policies or endorsements, either expressed or implied, of NIST, DOI/IBC, or the U.S. Government.

\section{References}

Yonatan Belinkov, Adam Poliak, Stuart M Shieber, Benjamin Van Durme, and Alexander M Rush. 2019. On adversarial removal of hypothesis-only bias in natural language inference. In Proceedings of the Eighth Joint Conference on Lexical and Computational Semantics (* SEM 2019), pages 256-262.

Daniel Borkan, Lucas Dixon, Jeffrey Sorensen, Nithum Thain, and Lucy Vasserman. 2019. Nuanced metrics for measuring unintended bias with real data for text classification. In Companion Proceedings of The 2019 World Wide Web Conference, pages 491-500. ACM.

Cornelia Caragea, Adrian Silvescu, and Andrea $\mathrm{H}$ Tapia. 2016. Identifying informative messages in disaster events using convolutional neural networks. In International Conference on Information Systems for Crisis Response and Management, pages 137147.

Dallas Card, Michael Zhang, and Noah A. Smith. 2019. Deep weighted averaging classifiers. In Proceedings of ACM FAT*

Rich Caruana. 1997. Multitask learning. Machine learning, 28(1):41-75.

Jacob Devlin, Ming-Wei Chang, Kenton Lee, and Kristina Toutanova. 2019. Bert: Pre-training of deep bidirectional transformers for language understanding. In Proceedings of the 2019 Conference of the North American Chapter of the Association for Computational Linguistics: Human Language Technologies, Volume 1 (Long and Short Papers), pages 4171-4186.

Yanai Elazar and Yoav Goldberg. 2018. Adversarial removal of demographic attributes from text data. In Proceedings of the 2018 Conference on Empirical Methods in Natural Language Processing, pages 1121.

Dumitru Erhan, Yoshua Bengio, Aaron Courville, and Pascal Vincent. 2009. Visualizing higher-layer features of a deep network.

Yaroslav Ganin, Evgeniya Ustinova, Hana Ajakan, Pascal Germain, Hugo Larochelle, François Laviolette, Mario Marchand, and Victor Lempitsky. 2016. Domain-adversarial training of neural networks. The Journal of Machine Learning Research, 17(1):2096-2030. 
Ian Goodfellow, Jean Pouget-Abadie, Mehdi Mirza, Bing Xu, David Warde-Farley, Sherjil Ozair, Aaron Courville, and Yoshua Bengio. 2014. Generative adversarial nets. In Advances in neural information processing systems, pages 2672-2680.

Alex Graves, Navdeep Jaitly, and Abdel-rahman Mohamed. 2013. Hybrid speech recognition with deep bidirectional lstm. In 2013 IEEE workshop on automatic speech recognition and understanding, pages 273-278. IEEE.

Starr Roxanne Hiltz, Jane A Kushma, and Linda Plotnick. 2014. Use of social media by us public sector emergency managers: Barriers and wish lists. In $I S$ CRAM.

Muhammad Imran, Prasenjit Mitra, and Carlos Castillo. 2016. Twitter as a lifeline: Human-annotated twitter corpora for nlp of crisis-related messages. In Proceedings of the Tenth International Conference on Language Resources and Evaluation (LREC 2016), Paris, France. European Language Resources Association (ELRA).

Diederik P Kingma and Jimmy Ba. 2014. Adam: A method for stochastic optimization. arXiv preprint arXiv:1412.6980.

Anna Kruspe. 2019. Few-shot tweet detection in emerging disaster events. arXiv preprint arXiv: 1910.02290 .

Jiwei Li, Xinlei Chen, Eduard Hovy, and Dan Jurafsky. 2016. Visualizing and understanding neural models in nlp. In Proceedings of NAACL-HLT, pages 681691.

Pengfei Liu, Xipeng Qiu, and Xuan-Jing Huang. 2017. Adversarial multi-task learning for text classification. In Proceedings of the 55th Annual Meeting of the Association for Computational Linguistics (Volume 1: Long Papers), pages 1-10.

Kaiji Lu, Piotr Mardziel, Fangjing Wu, Preetam Amancharla, and Anupam Datta. 2018. Gender bias in neural natural language processing. arXiv preprint arXiv:1807.11714.

Guoqin Ma. Tweets classification with bert in the field of disaster management

David Madras, Elliot Creager, Toniann Pitassi, and Richard Zemel. 2018. Learning adversarially fair and transferable representations. In International Conference on Machine Learning, pages 33843393.

Carleen Maitland, L Ngamassi, and Andrea Tapia. 2009. Information management and technology issues addressed by humanitarian relief coordination bodies. In Proceedings of the 6th International IS CRAM Conference. Gothenburg, Sweden.

Richard Mccreadie, Cody Buntain, and Ian Soboroff. 2019. Trec incident streams: Finding actionable information on social media.
Taro Miyazaki, Kiminobu Makino, Yuka Takei, Hiroki Okamoto, and Jun Goto. 2019. Label embedding using hierarchical structure of labels for twitter classification. In Proceedings of the 2019 Conference on Empirical Methods in Natural Language Processing and the 9th International Joint Conference on Natural Language Processing (EMNLP-IJCNLP), pages 6318-6323.

Robert Munro. 2011. Subword and spatiotemporal models for identifying actionable information in haitian kreyol. In Proceedings of the fifteenth conference on computational natural language learning, pages 68-77. Association for Computational Linguistics.

Graham Neubig, Yuichiroh Matsubayashi, Masato Hagiwara, and Koji Murakami. 2011. Safety information mining - what can nlp do in a disaster-. In Proceedings of 5th International Joint Conference on Natural Language Processing, pages 965-973.

Dat Tien Nguyen, Shafiq Joty, Muhammad Imran, Hassan Sajjad, and Prasenjit Mitra. 2016. Applications of online deep learning for crisis response using social media information. arXiv preprint arXiv:1610.01030.

Leysia Palen, Kenneth M Anderson, Gloria Mark, James Martin, Douglas Sicker, Martha Palmer, and Dirk Grunwald. 2010. A vision for technologymediated support for public participation \& assistance in mass emergencies \& disasters. ACM-BCS Visions of Computer Science 2010, pages 1-12.

Zhongyi Pei, Zhangjie Cao, Mingsheng Long, and Jianmin Wang. 2018. Multi-adversarial domain adaptation. In Thirty-Second AAAI Conference on Artificial Intelligence.

Jeffrey Pennington, Richard Socher, and Christopher D. Manning. 2014. Glove: Global vectors for word representation. In Empirical Methods in Natural Language Processing (EMNLP), pages 1532-1543.

Linda Plotnick, Starr Roxanne Hiltz, Jane A Kushma, and Andrea H Tapia. 2015. Red tape: Attitudes and issues related to use of social media by us countylevel emergency managers. In ISCRAM.

Samira Pouyanfar, Yudong Tao, Saad Sadiq, Haiman Tian, Yuexuan Tu, Tianyi Wang, Shu-Ching Chen, and Mei-Ling Shyu. 2019. Unconstrained flood event detection using adversarial data augmentation. In 2019 IEEE International Conference on Image Processing (ICIP), pages 155-159. IEEE.

Junaid Qadir, Anwaar Ali, Raihan ur Rasool, Andrej Zwitter, Arjuna Sathiaseelan, and Jon Crowcroft. 2016. Crisis analytics: big data-driven crisis response. Journal of International Humanitarian Action, 1(1):1-21.

Christian Reuter, Gerhard Backfried, Marc-André Kaufhold, and Fabian Spahr. 2018. Iscram turns 15: A trend analysis of social media papers 2004-2017. Proceedings of ISCRAM. 
Takeshi Sakaki, Makoto Okazaki, and Yutaka Matsuo. 2010. Earthquake shakes twitter users: real-time event detection by social sensors. In Proceedings of the 19th international conference on World wide web, pages 851-860.

Karen Simonyan, Andrea Vedaldi, and Andrew Zisserman. 2013. Deep inside convolutional networks: Visualising image classification models and saliency maps. arXiv preprint arXiv:1312.6034.

Jeannette N Sutton, Leysia Palen, and Irina Shklovski. 2008. Backchannels on the front lines: Emergency uses of social media in the 2007 southern california wildfires.

Andrea Tapia, Louis-Marie Ngamassi Tchouakeu, Edgar Maldonado, Kang Zhao, Harold Robinson, and Carleen Maitland. 2011a. Exploring barriers to coordination between humanitarian ngos: A comparative case study of two ngo's information technology coordination bodies. International Journal of Information Systems and Social Change, 2(2):1-25.

Andrea H Tapia, Kartikeya Bajpai, Bernard J Jansen, John Yen, and Lee Giles. 2011b. Seeking the trustworthy tweet: Can microblogged data fit the information needs of disaster response and humanitarian relief organizations. In Proceedings of the 8th International ISCRAM Conference, pages 1-10. ISCRAM Lisbon, Portugal.

Qizhe Xie, Zihang Dai, Yulun Du, Eduard Hovy, and Graham Neubig. 2017. Controllable invariance through adversarial feature learning. In Advances in Neural Information Processing Systems, pages 585596.

Brian Hu Zhang, Blake Lemoine, and Margaret Mitchell. 2018. Mitigating unwanted biases with adversarial learning. In Proceedings of the 2018 AAAI/ACM Conference on AI, Ethics, and Society, pages 335-340. ACM. 